\title{
Inflammatory cutaneous lesions and pulmonary manifestations in a new patient with autosomal recessive ISG15 deficiency case report
}

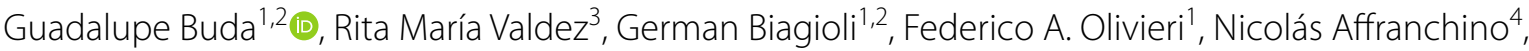 \\ Carolina Bouso ${ }^{5}$, Vanesa Lotersztein ${ }^{3}$, Dusan Bogunovic ${ }^{6,7,8}$, Jacinta Bustamante $9,10,11,12$ and Marcelo A. Martí ${ }^{*}$
}

\begin{abstract}
Interferon-stimulated gene 15 (ISG15) was the first ubiquitin-like modifier protein identified that acts by protein conjugation (ISGylation) and is thought to modulate IFN-induced inflammation. Here, we report a new patient from a non-consanguineous Argentinian family, who was followed for recurrent ulcerative skin lesions, cerebral calcifications and lung disease. Whole Exome Sequencing (WES) revealed two novel compound heterozygous variants (c.285del and c.299_312del, NM_005101.4 GRCh37(hg19), both classified as pathogenic according to ACMG criteria) in the ISG15 gene, resulting in a complete deficiency due to disruption of the second ubiquitin domain of the corresponding protein. The clinical phenotype of this patient is unique given the presence of recurrent pulmonary manifestations and the absence of mycobacterial infections, thus resulting in a phenotype distinct from that previously described in patients with biallelic loss-of-function (LOF) ISG15 variants. This case highlights the role of ISG15 as an immunomodulating factor whose LOF variants result in heterogeneous clinical presentations.
\end{abstract}

Keywords: Whole-exome sequencing, ISG15 gene, Case report, Ulcerative skin lesions, Lung disease

\section{To the Editor}

Interferon-stimulated gene 15 (ISG15) was the first ubiquitin-like modifier protein identified that acts by protein conjugation (ISGylation) [1]. It is one of the most upregulated genes upon Type I interferon treatment or pathogen infections, and its secreted form stimulates the production of cytokines and proliferation of NK cells in humans [2]. ISG15 is present in the gelatinase and secretory granules (but not in the azurophilic or

\footnotetext{
*Correspondence: marcelo.marti@gmail.com

1 Departamento de Química Biológica, Facultad de Ciencias Exactas y Naturales, Universidad de Buenos Aires (FCEyN-UBA) e Instituto de Química Biológica de la Facultad de Ciencias Exactas y Naturales (IQUIBICEN) CONICET, Pabellón 2 de Ciudad Universitaria, Buenos Aires, Argentina

Full list of author information is available at the end of the article
}

specific granules) of steady-state neutrophils, which release it upon bacterial challenge [3]. ISG15 is also secreted by many other cell types, including myeloid cells, acting as a very potent interferon gamma (IFN$\gamma$ ) inducing cytokine in lymphocytes (particular NK cells), and in synergy with IL-12. In humans, biallelic mutations in ISG15 were reported to result in an impaired IFN- $\gamma$ mediated immunity, leading to an increase predisposition to mycobacterial infections in most cases, as also observed in patients with Mendelian Susceptibility to Mycobacterial Diseases (MSMD) [4]; [5]; [6] (see Additional file 1: Table S2). Interestingly, ISG15-deficient patients usually present features of type 1 interferonopathy with cerebral calcifications; attributed to the role of ISG15 in controlling IFN-alpha/beta responses [8].

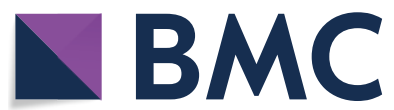

(c) The Author(s) 2020. This article is licensed under a Creative Commons Attribution 4.0 International License, which permits use, sharing, adaptation, distribution and reproduction in any medium or format, as long as you give appropriate credit to the original author(s) and the source, provide a link to the Creative Commons licence, and indicate if changes were made. The images or other third party material in this article are included in the article's Creative Commons licence, unless indicated otherwise in a credit line to the material. If material is not included in the article's Creative Commons licence and your intended use is not permitted by statutory regulation or exceeds the permitted use, you will need to obtain permission directly from the copyright holder. To view a copy of this licence, visit http://creativeco mmons.org/licenses/by/4.0/. The Creative Commons Public Domain Dedication waiver (http://creativecommons.org/publicdomain/ zero/1.0/) applies to the data made available in this article, unless otherwise stated in a credit line to the data. 
In the present work, we report a new patient with autosomal recessive (AR) complete ISG15 deficiency, followed for ulcerative skin lesions and lung disease. The patient was born in 2013 from a non-consanguineous Argentinean family. The brotherhood is completed with a healthy younger brother, and a healthy paternal stepbrother (Fig. 1a). She received BCG (Bacillus Calmette-Guérin) vaccination at birth. At 6 months of age, she presented with neck and axillary papuloulcerated lesions. At that time she was admitted at the hospital and received antibiotic treatment without clinical response, followed by spontaneous resolution 2 months later. Between 6 and 15 months of age, and due to recurrent skin ulcers, she was initially suspected of presenting BCG-itis. At 1 year old she showed novel similar lesions in the inguinal region and external genitalia, with bilateral and asymmetrical location and without response to either local or systemic treatments. The lesions showed slow healing ( 3 months) and the biopsy reported ulceration of dermis and epidermis, with necrosis of underlying hypodermis and without inflammatory reaction. Periodic acid-Schiff, acid-fast bacilli and Ziehl-Neelsen reactions were negative. Cultures for pyogenic bacteria, mycobacteria and fungi were also negative. Immunofluorescence of skin samples were negative for immunoglobulins (Ig) IgA, IgG, IgM and C3.

Starting at 2 years and 8 months of age, the patient began having respiratory manifestations including recurrent wheeze and lobar pneumonias (seven isolated and unrelated episodes over the span of 4 years). Three of them required hospitalization, antibiotics and oxygen therapy (for a period of 5 days on average); while one presented with images resembling necrotizing pneumonia with negative microbiological tests. She was kept asymptomatic between episodes. Lung and chest computed tomography (CT) scanning (Fig. 2a) showed some non specific and residual lesions, while brain CT evidenced basal ganglia calcifications (Fig. 2b). Due to the pulmonary manifestations, mycobacterial infection was suspected again. However, clinical inspection by the attending physician discarded BCG-itis, since: (i) no mycobacteria species (or other microorganisms) were isolated from either skin biopsies, or from multiple bronchoalveolar lavages (BAL); (ii) the skin lesions healed without the use of any antimycobacterial treatment, and (iii) the pulmonary manifestations, although seemed

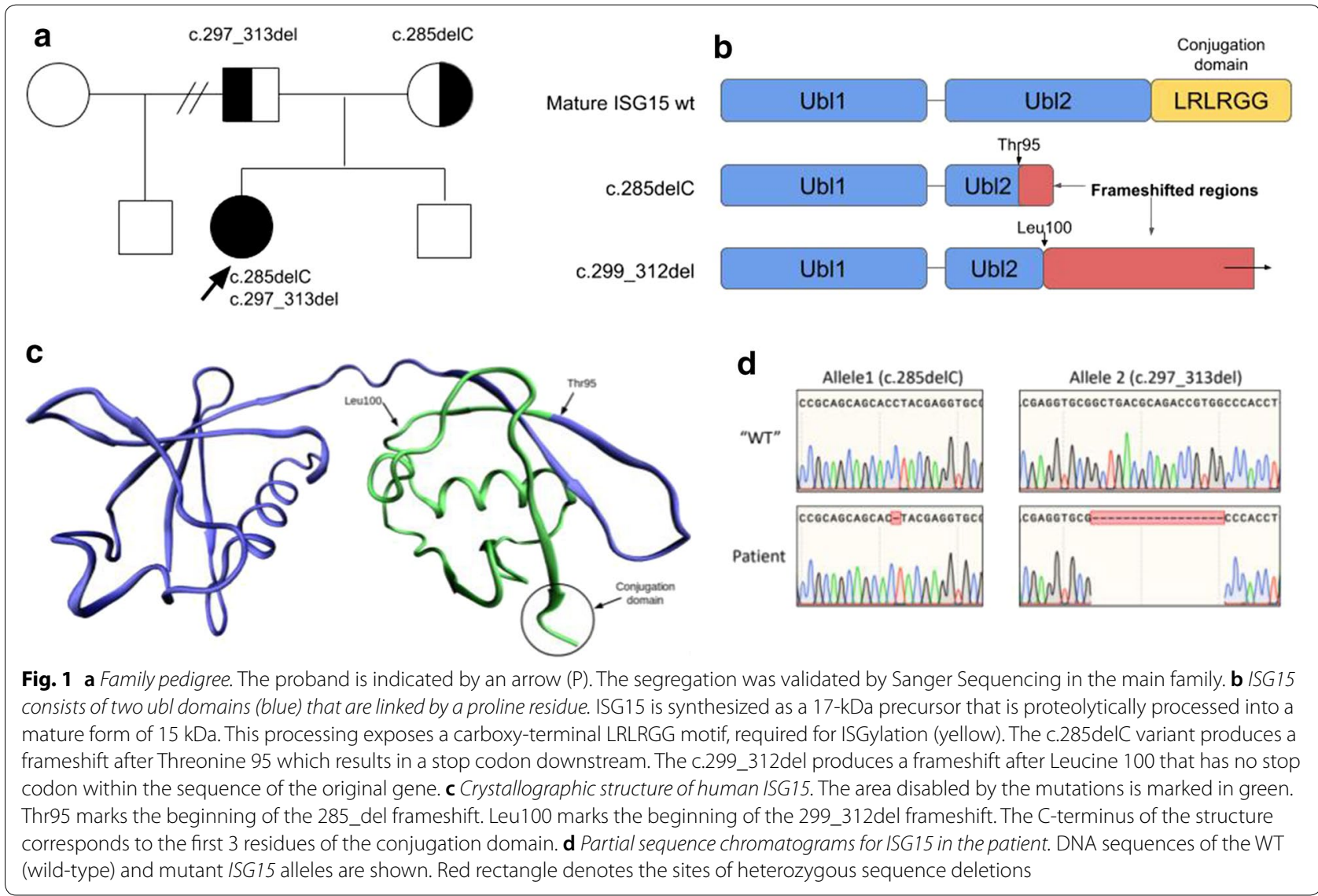


a

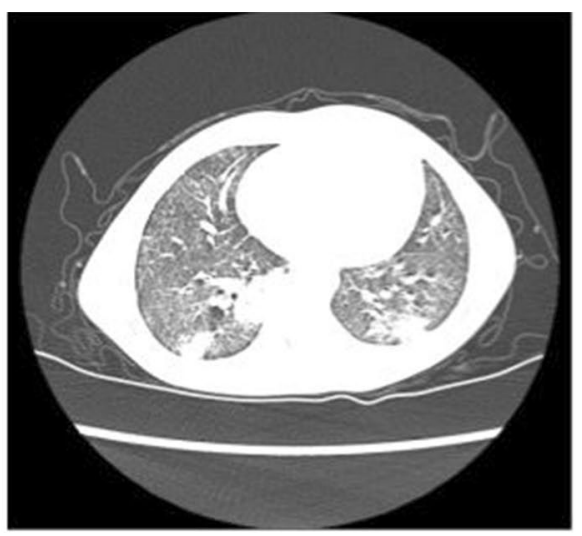

b

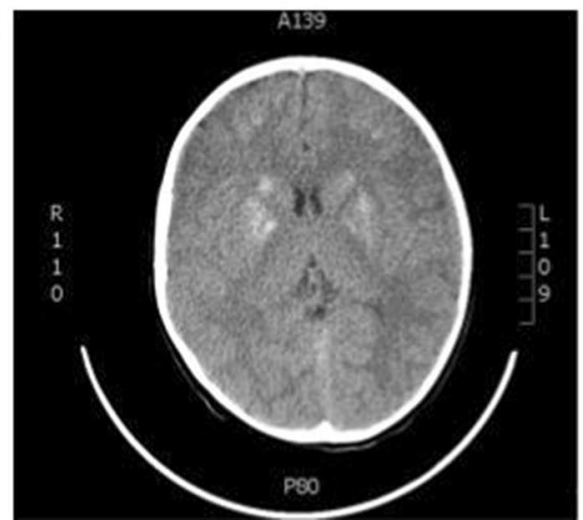

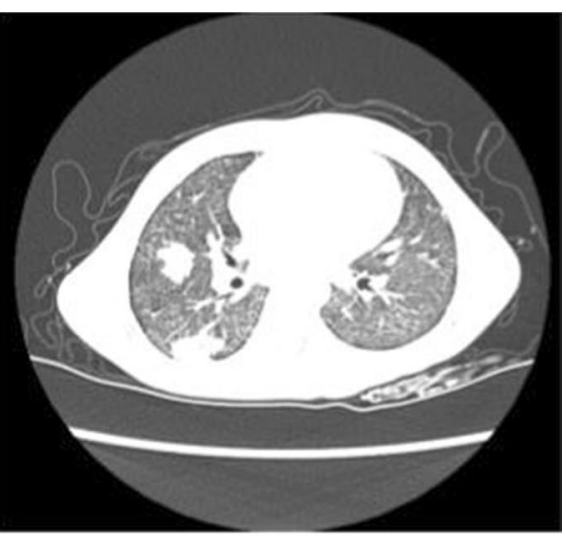

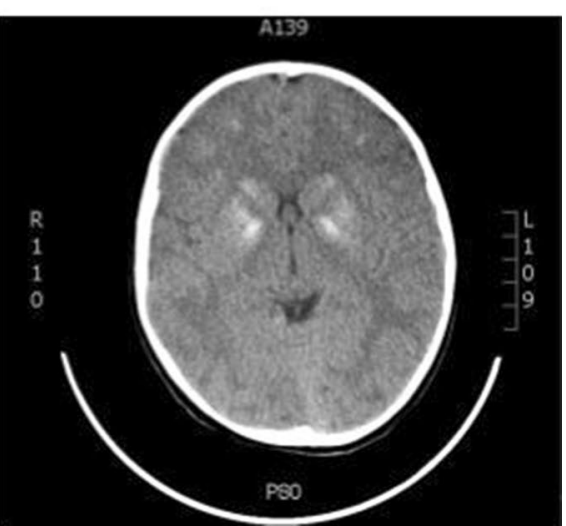

Fig. 2 a Chest CT scan. Bilateral focal areas of parenchymal consolidations, some of them with air bronchograms and with nodular appearance, distributed in the peripheral portions of the lower zones. Bilateral widespread septal thickening and peripheral tree in-bud changes in both lungs is observed too. Lobar air-trapping (ILL) on expiration was present. b Brain CT scan. Calcifications at the level of the caudate nuclei, lenticular nuclei and subcortical region of both frontal lobes are shown in the images

progressive, were presented in multiple episodes affecting different sections of the lung parenchyma.

Laboratory tests showed elevated acute-phase reactants, mainly the erythrocyte sedimentation rate. Number and percentages of T, B and NK cells showed to be in adequate range for the age, as well as Igs, C3 and $\mathrm{C} 4$. In addition, inadequate antibody response to some protein antigens and polysaccharides was found (Additional file 3: Table S3). The dihydrorhodamine (DHR) assay on neutrophils was normal. Autoimmune workup revealed transient positive lupus anticoagulant and non specific ASMA auto-Ab. Due to the patient multisystemic picture affecting predominantly the skin (chronic and recurrent ulcers) and the lower respiratory tract (lung disease associated with hypoxia and persistent lesions despite adequate treatment), the patient was initially suspected to carry an autoinflammatory disease. Currently, the patient is 7 years old and presents height and weight appropriate for her age. Healing lesions are still present in retroauricular regions, cervical, bilateral axillary, bilateral inguinal and in labia majora, with associated lipoatrophy. She shows delayed and peculiar dentition with the absence of canines and no other abnormalities. Additionally, she displays chronic lung disease with hypoxemic chronic respiratory failure and is being treated with oxygen therapy at home $(0.5 \mathrm{~L} / \mathrm{min}$ in sleep and $1 \mathrm{~L} / \mathrm{min}$ during the day).

Whole Exome Sequencing (WES) was performed and a total of 100,341 variants were obtained. The first analysis was focused on 55 autoinflammatory genes (Additional file 1: Table S1), but no clinical significant variants were found. However, two clinical relevant heterozygous variants in the ISG15 gene (see Additional file 1: Fig. S1), which codifies for an Ubiquitin-like protein (termed Ubiquitin like modifier ISG15), were identified. The first heterozygous variant c.285del (NM_005101.4, GRCh37(hg19)), also known as c.284del, corresponds to a single nucleotide deletion that is predicted to cause a frameshift in exon 2 (p.Tyr96Thrfs*5) and produces the gain of a stop codon 5 residues downstream from the variant. The second heterozygous variant, c.299_312del (NM_005101.4, GRCh37(hg19)), also known as c.297_313del, corresponds 
to a 14 nucleotide deletion in exon 2 which is also predicted to produce a frameshift below residue Leu100; p.Leu100Argfs (Fig. 1b). Sanger sequencing and familial segregation were performed confirming that the patient is a compound heterozygous for the above described mutations in ISG15 gene (Fig. 1d). The parents were each heterozygous for one of the reported variants, and the younger brother does not present any of them. All data is consistent with an AR mode of inheritance of ISG15 deficiency (Fig. 1a). According to ACMG criteria [7], both variants follow the pathogenic criterion: pathogenic very strong (PVS1), since they are both null variants, pathogenic moderate 2 (PM2) due to their low frequencies (with no reports or very low allele frequency in population databases such as GnomAD); and pathogenic moderate 3 (PM3), since we confirmed by Sanger Sequencing that the patient inherited both variants in trans, each from one parent. Moreover, it was reported that both variants result in loss of expression and LOF (PS3). To accomplish that, Dusan and col. used an in vitro overexpression system in which both variant carrying alleles were compared with the wild type form of ISG15 [8] .

The ISG15 protein is composed of two tandem repeats of ubiquitin-like domains, the $\mathrm{N}$-terminal domain is required for the efficient E3 ligase-mediated transfer of ISG15 from the E2 enzyme UbCH8 to its substrates, although it is dispensable in the activation and transthiolation steps [9]. The two ubiquitin domains of ISG15 play different critical roles in ISGylation, which is unique among ubiquitin and ubiquitin-like modifiers. Both frameshifts variants clearly disrupt the second domain, causing the loss of the $\mathrm{C}$-terminus ending that contains a very conserved amino acid sequence Leu Arg Leu Arg Gly Gly (LRLRGG), corresponding to the ISGylation site and thus triggering the loss of function of the entire protein [10] (Fig. 1b, c). Compared with ubiquitin, which has very high (close to $100 \%$ identity) cross-species conservation, ISG15 protein has relatively low cross-species conservation. Its absence in many eukaryotic species suggests that it is not an essential housekeeping gene, leaving more room for its diversification during evolution. Pathogenic variants in ISG15 gene are a recently defined cause of type I human interferonopathy [11]. Biallelic loss-of-function variants in ISG15 were initially described in the context of patients with Immunodeficiency 38 (OMIM: 616126), an AR disorder which confers syndromic Mendelian susceptibility to Mycobacterial diseases (MSMD) and affects about 1 in 50,000 individuals exposed to environmental mycobacteria or BCG vaccine strains [4]. Surprisingly, and despite the antiviral functions of ISG15 described in mice, patients described to date do not present viral susceptibility phenotype.
The ISG15-IFN- $\gamma$ circuit operates principally between granulocytes and NK cells [4, 12]. Elevated ISG expression and cerebral calcification are consistent features in patients with biallelic pathogenic variants in ISG15, with a strong neuroradiological overlap with patients with Aicardi-Goutières syndrome (AGS) [13.]

STING-associated vasculopathy with onset in infancy (known as SAVI syndrome) is an early-onset systemic inflammatory phenotype characterized by severe cutaneous vasculopathy and major interstitial lung disease, caused by enhanced sensitivity or ligandindependent (constitutive) activation of a non-nucleic acid receptor component (for example, an adaptor molecule) of the IFN-induced signalling pathway, such as in the case of de novo and inherited pathogenic variants in TMEM173 gene, which encodes the protein STING (stimulator of interferon genes) [14, 15].

Here, we report a patient that clinically resembles a less severe auto-inflammatory disease, with lung and skin involvement, similar to those observed in other interferonopathies such as SAVI, showing recurrent slow healing skin ulcers and respiratory manifestations, that do not respond adequately to antibiotic treatment nor allow identification of a particular infectious agent. On the other hand, the CT scan of her brain shows basal ganglia calcifications, a common feature present in some autoinflammatory diseases like Chronic Atypical Neutrophilic Dermatosis with Lipodystrophy and Elevated Temperature (CANDLE) and that are most prominent in AGS, but that were also well characterized in patients with Immunodeficiency 38. WES revealed the presence of two protein-truncating pathogenic variants in trans in the ISG15 gene, both of which eliminate the second ubiquitin-like domain and cause LOF variants.

Our work underscores the strength of WES approach to diagnose immune related disorders with molecular precision, even for isolated (non-familial) cases, leading to the extension of the reported phenotype which now includes recurrent lung disease and cutaneous inflammatory manifestations, without mycobacterial infections. Our findings also highlight the role of ISG15 as an immunomodulating factor whose LOF mutations result in clinical heterogenous presentations. However it should be noted that the significance of the observed genotype to phenotype association could be limited since we are analyzing a single case. Finally, concerning the molecular mechanism leading to disease, we hypothesize that there might be a factor such as infection, stress or vaccination that may trigger the inflammatory manifestations of the observed multisystemic disease, that initially appeared with skin ulcerations and continued with pulmonary affectation. 


\section{Supplementary information}

Supplementary information accompanies this paper at https://doi. org/10.1186/s13223-020-00473-7.

Additional file 1: Table S1. Primary genetic panel studied. Genes related to monogenic autoinflammatory diseases.

Additional file 2: Table S2. Patients reported to date with ISG15 deficiency, including ours. IBGC refers to idiopathic basal ganglia calcification

Additional file 3: Table S3. Immune evaluation results. ${ }^{*} 25$ th and 75 th percentiles of age-related normal values for lymphocyte subpopulations. Cellular immunology laboratory of "Pediatric Hospital Prof. Dr. Juan P. Garrahan". + Reference values for serum IgA, IgG and IgM according to age, expressed as median \pm SD. Ref: Stiehm ER, Fudenberg HH. Serum levels of Immune Globulins in health and disease. Pediatrics 37: 715, 1966.

Additional file 4: Figure S1. Sequence alignment view of the BAM file. The two deletions are shown as lines.

\section{Acknowledgements}

The authors thank the case family for their participation in this study and all the health care professionals that sent medical information and participate in this case report, in particular Silvia Danielian, Mariana Villa and Matias Oleastro (Immunology), Bettina Cervini (Dermatology) and Claudio Castaños (Pneumonology) from the Garrahan Hospital, Buenos Aires, Argentina, Dr. Julio Cesar Orellana (Immunology) from Cordoba Children's Hospital, and Margarita Larralde (Dermatology) from the German Hospital, who has extensively studied and treated the patient during all this years and provided professional opinion and material.

\section{Authors' contributions}

GB analyzed and interpreted the patient data (WES) regarding the disease, drafted the work and was a major contributor in writing the manuscript. RMV geneticist, followed the patient, apply the patient to the PAMPA Project and provided material and relevant clinical information. VL geneticist, followed the patient and provided material and relevant clinical information. NA pediatrician, currently follows the patient and provided material and relevant clinical information. CB currently does the immunological follow-up of the patient and provided material and relevant clinical information. FAO did the bioinformatic analysis of the variants based on the Crystallographic structure of human ISG15, designed and added some of the figures related to the impact of the variants in the tridimensional structure of the protein. JB analyzed the family segregation of the variants by Sanger sequencing, drafted the work and substantively revised it. DB analyzed the family segregation of the variants by Sanger sequencing, drafted the work and substantively revised it. MM analyzed and interpreted the patient data (WES) regarding the disease, have drafted the work and substantively revised it. All authors read and approved the final manuscript.

\section{Funding}

This present work was partially supported by Novartis PAMPA Project (Argentine Precision Program in Autoinflammatory Diseases) in collaboration with Bitgenia (https://apps.bitgenia.com/pampa/) and by the National Institute of Allergy and Infectious Diseases (5R37Al095983) from the National Institutes of Health and grant from the French National Research Agency (ANR) under GENMSMD (ANR-16-CE17.0005-01).

\section{Availability of data and materials}

All data generated or analysed during this study are included in this published article [and its supplementary information files].

\section{Ethics approval and consent to participate}

Written informed consent for the participation in the PAMPA Project was obtained from the patient's parents.

\section{Consent for publication}

Written informed consent for publication of clinical details was obtained from the patient's parents. We confirm that we have read the Journal's position on issues involved in ethical publication and affirm that this work is consistent with those guidelines.

\section{Competing interests}

The authors declare that they have no competing interests.

\section{Author details}

${ }^{1}$ Departamento de Química Biológica, Facultad de Ciencias Exactas y Naturales, Universidad de Buenos Aires (FCEyN-UBA) e Instituto de Química Biológica de la Facultad de Ciencias Exactas y Naturales (IQUIBICEN) CONICET, Pabellón 2 de Ciudad Universitaria, Buenos Aires, Argentina. ${ }^{2}$ Bitgenia, Buenos Aires, Argentina. ${ }^{3}$ Hospital Militar Central, Servicio de Genética, Buenos Aires, Argentina. ${ }^{4}$ Hospital Juan P. Garrahan, Servicio de Pediatría, Buenos Aires, Argentina. ${ }^{5}$ Hospital Juan P. Garrahan, Servicio de Inmunología y Reumatología, Buenos Aires, Argentina. ${ }^{6}$ Department of Microbiology, Icahn School of Medicine at Mount Sinai, New York, USA. ${ }^{7}$ Department of Pediatrics, Icahn School of Medicine at Mount Sinai, New York, USA. ${ }^{8}$ The Mindich Child Health and Development Institute, Icahn School of Medicine at Mount Sinai, New York, USA. ${ }^{9}$ Laboratory of Human Genetics of Infectious Diseases, Necker Branch, INSERM, Paris, France. ${ }^{10}$ Laboratory of Human Genetics of Infectious Diseases, Necker Branch, INSERM, University of Paris, Imagine Institute, Paris, EU, France. ${ }^{11}$ St. Giles Laboratory of Human Genetics of Infectious Diseases, Rockefeller Branch, The Rockefeller University, New York, USA. ${ }^{12}$ Study Center of Immunodeficiencies, Necker Hospital for Sick Children, Paris, EU, France.

Received: 16 January 2020 Accepted: 6 August 2020

Published online: 03 September 2020

\section{References}

1. Zhao C, Denison C, Huibregtse JM, Gygi S, Krug RM. Human ISG15 conjugation targets both IFN-induced and constitutively expressed proteins functioning in diverse cellular pathways. Proc Natl Acad Sci U S A. 2005;102(29):10200-5.

2. D'Cunha J, Knight E Jr, Haas AL, Truitt RL, Borden EC. Immunoregulatory properties of ISG15, an interferon-induced cytokine. Proc Natl Acad Sci U S A. 1996;93(1):211-5

3. Bogunovic D, Boisson-Dupuis S, Casanova J-L. ISG15: leading a double life as a secreted molecule. Exp Mol Med. 2013;12(45):e18.

4. Bogunovic D, Byun M, Durfee LA, Abhyankar A, Sanal O, Mansouri D, et al. Mycobacterial disease and impaired IFN-y immunity in humans with inherited ISG15 deficiency. Science. 2012;337(6102):1684-8.

5. Bustamante J, Boisson-Dupuis S, Abel L, Casanova J-L. Mendelian susceptibility to mycobacterial disease: genetic, immunological, and clinical features of inborn errors of IFN- $\gamma$ immunity. Semin Immunol. 2014;26(6):454-70

6. Rosain J, Kong X-F, Martinez-Barricarte R, Oleaga-Quintas C, Ramirez-Alejo N, Markle J, et al. Mendelian susceptibility to mycobacterial disease: 2014-2018 update. Immunol Cell Biol. 2019;97(4):360-7.

7. Richards S, Aziz N, Bale S, Bick D, Das S, Gastier-Foster J, et al. Standards and guidelines for the interpretation of sequence variants: a joint consensus recommendation of the American College of Medical Genetics and Genomics and the Association for Molecular Pathology. Genet Med. 2015;17(5):405-24.

8. Martin-Fernandez M, Bravo García-Morato M, Gruber C, Murias Loza S, Malik MNH, Alsohime F, et al. Systemic type I IFN inflammation in human ISG15 deficiency leads to necrotizing skin lesions. Cell Rep. 2020;31(6):107633.

9. Chang Y-G, Yan X-Z, Xie Y-Y, Gao X-C, Song A-X, Zhang D-E, et al. Different roles for two ubiquitin-like domains of ISG15 in protein modification. J Biol Chem. 2008;283(19):13370-7.

10. Zhang $D$, Zhang D-E. Interferon-stimulated gene 15 and the protein ISGylation system. J Interferon Cytokine Res. 2011;31 (1):119-30.

11. Zhang X, Bogunovic D, Payelle-Brogard B, Francois-Newton V, Speer SD, Yuan C, et al. Human intracellular ISG15 prevents interferon-a/ $\beta$ overamplification and auto-inflammation. Nature. 2015;517(7532):89-93.

12. Trinchieri G. Interleukin-12 and the regulation of innate resistance and adaptive immunity. Nat Rev Immunol. 2003;3(2):133-46.

13. Crow YJ, Manel N. Aicardi-Goutières syndrome and the type I interferonopathies [Internet]. Vol. 15, Nature Reviews Immunology. 2015. p. 429-40. http://dx.doi.org/10.1038/nri3850. 
14. Liu Y, Jesus AA, Marrero B, Yang D, Ramsey SE, Sanchez GAM, et al. Activated STING in a vascular and pulmonary syndrome. N Engl J Med. 2014;371(6):507-18

15. Jeremiah N, Neven B, Gentili M, Callebaut I, Maschalidi S, Stolzenberg M-C, et al. Inherited STING-activating mutation underlies a familial inflammatory syndrome with lupus-like manifestations. J Clin Invest. 2014;124(12):5516-20.

\section{Publisher's Note}

Springer Nature remains neutral with regard to jurisdictional claims in published maps and institutional affiliations.
Ready to submit your research? Choose BMC and benefit from:

- fast, convenient online submission

- thorough peer review by experienced researchers in your field

- rapid publication on acceptance

- support for research data, including large and complex data types

- gold Open Access which fosters wider collaboration and increased citations

- maximum visibility for your research: over 100M website views per year

At BMC, research is always in progress.

Learn more biomedcentral.com/submissions 